\title{
Protokoll der Generalversammlung der ÖGUM 2013
}

\author{
Freitag, 11. Oktober, 2013, 17:10 - 18:15; Internationales \\ Congress Center Stuttgart (ICS)
}

Anwesend: H. Steiner, A. Klauser, C. Kollmann, E. Hafner, D. Kerö, H. Kathrein, E. Prosch, W. Eppel, A. Brichta, H. Gregor, S. Meng, C. Aiginger, T. Ybinger, A. Sachs, K. Maurer, T. Scholler, D. Koppin, B. Pertl, H. Harlass, P. Tschak, E. Krampl-Bettelheim, K. Anzböck, M. Metzenbauer, C. Eichler, J. Deutinger, G. Schramm-Marhardt, H. Musil, G. Faltl, G. Bräutigam, W. Richter, M. Schamp, M. Riccabona, B. Ploder, M. Häusler, C. Siedentopf, R. Mathies, A. Hollerweger (37)

ÖGUM Office: B. Kasperak, D. Zizka (nicht stimmberechtigt)

1. Begrüßung und Feststellung der Beschlussfähigkeit.
H. Steiner begrüßt die Teilnehmer. Beschlussfähigkeit wird festgestellt.

2. Genehmigung des Protokolls der Generalversammlung vom 28. 09. 2012 Das Protokoll wird einstimmig genehmigt.

3. Bericht des Präsidenten H. Steiner berichtet, dass die ÖGUM eine wachsende Gesellschaft ist. Der derzeitige Mitgliederstand beträgt 928. Es soll die Zahl 1000 erreicht werden. H. Steiner informiert die Anwesenden über die Vorstandsitzungen und die erweiterte Vorstandssitzung in Wien. Diese inkludieren auch die Arbeitsgruppen. Er berichtet über die enge Zusammenarbeit mit den 
Schwesterngesellschaften, DEGUM und SGUM. Er betont die wichtige Zusammenarbeit mit der EFSUMB bezüglich der gemeinsamen europäischen Ausbildungsstandards und der internationalen Vernetzung. G. Mathis ist auf eigenen Wunsch aus dem Education and Professional Standards COMMITTEE/EFSUMB ausgeschieden. Er bedankt sich für dessen Mitarbeit und sein großes Engagement. Die ÖGUM wird mit H. Prosch in diesem Komitee weiterhin vertreten sein. C. Kollmann ist der österreichische Vertreter im European Committee for medical ultrasound safety ECMUS).

4. Die ÖGUM Website hat ein Relaunch erfahren. Sie ist anwenderfreundlich und schneller geworden. Die Arbeitskreise werden ihre Informationen aktualisieren und das Layout wird vereinheitlicht. Auf der Website wurde die Kursleiterliste auf den neusten Stand gebracht. Die Kursleiter werden von der Geschäftsstelle angeschrieben und um den Nachweis ihrer Aktivität gebeten. Nach Auswertung der eingesandten Informationen werden sie auf der Website auf inaktiv gesetzt oder entfernt.

Ein Teilnehmer fragt, ob der Preis für das „Ultraschall in der Medizin“ sowie bei der DEGUM auch erhöht wird. H. Steiner informiert, dass er noch keine diesbezügliche Information vom Thieme Verlag erhalten hat. $\mathrm{H}$. Steiner fragt, ob eine gedruckte Ausgabe der Zeitschrift „Ultraschall in der Medizin" in der heutigen Zeit überhaupt noch nötig ist. Es soll dazu eine Mitgliederbefragung stattfinden, wenn eine Vertragsverlängerung bzw. -adaptierung ansteht. Riccabona weist darauf hin, dass der Impact Faktor bei einer geringeren Auflage der Druckversion sinken könnte. Die Geschäftsstelle soll diesbezüglich Informationen einholen. Riccabona fragt an, ob es möglich ist, dass die Arbeitskreise in Anlehnung an die Landesgruppen eigene Konten haben können, um Fortbildungen zu finanzieren. H. Steiner antwortet, dass eine Strukturadaptierung überlegt werden könnte, aber die ÖGUM bzw. der Vorstand voll haftbar und verantwortlich ist. Ebenso müssen lt. Vereinsrecht einige Vorgaben berücksichtigt werden. A. Klauser berichtet über das Dreiländertreffen 2014 in Innsbruck und informiert das Publikum über das verkürzte Treffen.
5. Kassabericht (C. Kollmann) Kollmann berichtet über die Einnahmen. Er weist auf die Rekordzahl der Mitglieder und die dadurch gestiegenen Einnahmen der Mitgliedsgebühren hin. Die Zahlungsmoral ist mit 96,2 \% ausgezeichnet. Weitere Einnahmen wurden aus dem Verkauf von Plaketten, Poster und von den Landesgruppen akquiriert. Er berichtet über einen Verlust im Abrechnungsjahr 2012. Dieser erklärt sich aus den hohen Kosten für das Thieme Abo, durch Verrechnung der Infrastrukturkosten $(2011,2012)$, C. Kollmann informiert, dass die aktuellen Mitgliedsgebühren beibehalten werden. Die 1000er Schallmauer soll durch verstärkte Mitgliederwerbung durchbrochen werden.

6. Entlastung des Kassiers und des Vorstandes

H. Prosch als Rechnungsprüfer der ÖGUM bestätigt die korrekte Finanzgebarung des Vereins ÖGUM nach dem österreichischen Vereinsgesetz für den Prüfungszeitraum 01.01. 31.12.2012 sowie die ordnungsgemäße Verwendung der Vereinsmittel laut Vereinsstatuten von 2006. Die Belege wurden stichprobenartig geprüft. Er stellt den Antrag auf Entlastung des Kassiers, welcher einstimmig angenommen wird. Es gibt keine Enthaltungen. H. Steiner bedankt sich bei den Rechnungsprüfern.

T. Meng stellt den Antrag auf Entlastung des Vorstandes, welcher einstimmig angenommen wird. Es gibt keine Enthaltungen. Im Namen des Vorstandes bedankt sich H. Steiner für das entgegengebrachte Vertrauen. H. Steiner teilt mit, dass 2 neue Rechnungsprüfer gewählt werden müssen, da P. Schwärzler nun in Hamburg/DE und H. Prosch ein kooptiertes Mitglied des ÖGUM Vorstands ist. Es werden M. Metzenbauer und T. Ybinger einstimmig gewählt.

7. Bericht der Landesgruppen Es wurden Berichte vorgetragen und abgegeben von Vorarlberg (Kurskosten für Ausbildungsärzte von ÖGUM zertifizierten Kursen werden vom Krankenanstaltenverbund übernommen!), Tirol, Salzburg, Niederösterreich und Wien. Berichte von Oberösterreich, Burgenland, Steiermark und Kärnten werden von der Geschäftsstelle nachgefordert.

8. ÖGUM Förderpreis 2013 H. Steiner begrüßt C. Siedentopf, Ge- winner des ÖGUM Förderpreises 2013. C. Siedentopf hält seinen Vortrag über: Meralgia paraesthetica: Ultraschall gezielte Infiltration mit Follow-up-Daten über 12 Monate.

9. Neue Website (C. Kollmann)

C. Kollmann berichtet über die Erneuerung der Website. 90\% der Inhalte sind bereits online. Der Provider wurde gewechselt, damit die Nutzer schneller zu den gewünschten Informationen gelangen. Die Arbeitskreise werden aufgefordert ihre Inhalte zu aktualisieren und die Ausbildungsrichtlinien einheitlich zu gestalten. Die Website soll vermehrt die Studenten und Ärzte in Ausbildung ansprechen (4students, Kurskalender, ...). Die Geschäftsstelle wird die Kursleiter/innen anschreiben um über die Online Kursanmeldung zu informieren. Kollmann berichtet über das Member Login, welches Ende des Jahres online gehen soll. In diesem Bereich können die Plaketten online bestellt werden. Ebenso können die Poster in bester Auflösung runtergeladen werden. Kollmann wird Informationen über Gerätetechnik zur Verfügung stellen. Alle Arbeitskreise können und sollen Materialien zur Verfügung stellen. Die Geschäftsstelle wird die Informationen sammeln und in den Member Login-Bereich einarbeiten.

10.Allfälliges

H. Steiner fragt die Teilnehmer was ihnen in Stuttgart gefällt und bittet um Verbesserungsvorschläge für US 2014 in Innsbruck. Folgende Vorschläge werden A. Klauser (Kongresspräsidentin) und B. Kasperak (Kongresssekretariat) mitgeteilt.

- lange Wartezeiten im Medien Center, zu wenig Personal, große Probleme bei Videos.

- unnötige und die Teilnehmer verärgernde Kongresseinteilung, wie z.B. am Freitag Nachmittag 3 gynäkologische Sitzungen parallel, am Vormittag dagegen fast kein Programm, und kein pränatales Programm. - angiologische Sitzungen parallel Vorschlag: R. Mathies bekommt das Programm für 2014 zur Kontrolle, um Überschneidungen zu vermeiden. - Tickets für Gesellschaftsabend waren zu schnell ausverkauft - es soll in Innsbruck sichergestellt werden, dass genügend Tickets für die Abendveranstaltung vorhanden sind.

- zu wenig Terminals, um E-mails zu 
überprüfen.

- keine Möglichkeit Essen zu kaufen . - es soll ein Lunch für die Teilnehmer der Anwenderseminare (Mittwoch) angeboten werden.

A. Klauser und H. Steiner versprechen für das DLT Innsbruck, dass es gut funktionieren wird (Medienannahmestelle, keine Überschneidungen im Programm, Essensversorgung,..) da die Kongressorganisation seit dem
Jahr 2000 immer dieselbe und auf diese Probleme vorbereitet ist. Ebenso ist die Firma ESTENSIS (zuständig für die Technik und Medien-Center) seit Beginn dabei und ist auf Videos vorbereitet und wird mit genügend Personal vor Ort sein.

gezeichnet der ÖGUM Präsident, der ÖGUM Sekretär 